\title{
Addressing the importance of melanoma tumor-infiltrating lymphocytes in disease progression and clinicopathological characteristics
}

\author{
TATJANA ZABLOCKA ${ }^{1-4}$, ANNA NIKOLAJEVA ${ }^{1}$, MADARA KREISMANE ${ }^{5}$, \\ DACE PJANOVA $^{5}$ and SERGEJS ISAJEVS ${ }^{1,2,4}$
}

\author{
${ }^{1}$ Department of Pathology, Faculty of Medicine, University of Latvia, LV-1004 Riga; ${ }^{2}$ Riga East University Hospital, \\ Centre of Pathology, LV-1038 Riga; ${ }^{3}$ Pauls Stradins Clinical University Hospital, Institute of Pathology, LV-1002 Riga; \\ ${ }^{4}$ Department of Microbiology and Pathology, Hospital of Traumatology and Orthopaedics, LV-1005 Riga; \\ ${ }^{5}$ Latvian Biomedical Research and Study Centre, LV-1067 Riga, Latvia
}

Received March 11, 2021; Accepted August 27, 2021

DOI: $10.3892 / \mathrm{mco} .2021 .2417$

\begin{abstract}
Tumor-infiltrating lymphocytes (TILs) in primary cutaneous melanoma are considered to represent the host's antitumor immunological response; however, whether there are associations between TIL grade and histopathological characteristics and disease survival remains controversial. BRAF mutational status has been established as a routine screening method in advanced malignant melanoma, and worse prognosis rates have been demonstrated in patients harboring BRAF mutations. However, the general impact of BRAF mutational status on survival and histopathological characteristics is still debated. The aim of the present study was to compare the value of the assessment of TIL grade in stages I-II nodular and superficial spreading melanoma and BRAF mutational status, and its influence on clinicopathological characteristics. Altogether, 85 patients at stage IA-IIC who underwent melanoma surgical treatment at the Riga East University Hospital between 2012 and 2017 were retrospectively enrolled in the study. The histopathological characteristics were assessed according to the current World Health Organization and The American Joint Committee on Cancer 8th edition guidelines. The current study showed that patients with melanoma with high TIL grade had significantly better progression-free survival than patients with low TIL grade (hazard ratio, 4.9; 95\% CI, 2.3-11.2; P<0.0001). BRAF mutations were observed in 52 patients $(61.2 \%)$. BRAF mutational status in melanoma was associated with Clark invasion level $(\mathrm{P}=0.045)$, patient age $(\mathrm{P}=0.02)$ and TIL $(\mathrm{P}=0.04)$. The assessment of TIL grade
\end{abstract}

Correspondence to: Dr Tatjana Zablocka, Department of Pathology, Faculty of Medicine, University of Latvia, 3 Jelgavas Street, LV-1004 Riga, Latvia

E-mail: tatjana.zablocka@gmail.com

Key words: melanoma, histopathology, tumor infiltrating lymphocytes, BRAF V600 in stage I-II melanoma demonstrated prognostic significance value and may help improve risk assessment in the future.

\section{Introduction}

Melanoma is a tumor with high impact through its rapidly growing incidence, high mortality, increased complexity, and high care costs in advanced stages (1). Melanoma incidence has been increasing in all European regions and the USA for the last few decades (2). The most well-established criteria's of melanoma prognosis are the tumor thickness and invasion, which are strongly associated with metastatic capacity. These features are the most established predictors of mortality at diagnosis (2-5).

There are four different major subtypes of melanoma, including superficial spreading (60\%-70\%), nodular (15\%-30\%), lentigo maligna (10\%), and acral lentiginous (5\%). Other rare variants of melanoma include desmoplastic, spitzoid melanoma within blue nevus or congenital nevus, pigmented epithelioid melanocytoma, and other variants (6). Current World Health Organization (WHO) classification of skin tumors subdivided melanoma based on solar elastosis assessed by dermal elastic fibers to measure cumulative sun damage $(7,8)$. Melanoma diagnostic biomarkers can be categorized into five groups, including visual, histopathological, morphological, immunohistochemical, and serological/molecular biomarkers $(1,4)$. Currently, the most reliable method for the diagnosis and prognosis of malignant melanoma is histopathological examination (8).

Tumor-infiltrating lymphocytes (TIL) are considered a manifestation of the host immune response to the tumor (9). It has been previously shown that TIL grade is an independent predictor of survival and sentinel lymph node (SLN) status in melanoma patients. Patients with a pronounced TIL infiltrate have better prognosis (9). Furthermore, a higher level of TIL in primary invasive melanoma samples is associated with a lower risk of death due to the malignancy (10-12). Recent breakthroughs in tumor immunotherapy such as immune checkpoint blockade implementing antibodies like cytotoxic T lymphocyte-associated protein-4 (CTLA-4) and 
programmed cell death protein-1 (PD-1) as well as its ligand PD-L1 $(13,14)$ have demonstrated the role of immune mechanisms in the pathogenesis and progression of this disease (15). Moreover, the numbers, localization, and phenotypes of TIL are predictive of the response to the immunotherapy and essential modulators of disease progression (13-15).

Recent genomic classification subdivides melanoma into four subtypes based on the pattern of the most prevalent significantly mutated genes: mutant BRAF, RAS, NF1, and triple-WT (wild-type) (16). BRAF V600 mutations were recognized as the primary driver mutation in tumor progression of melanoma patients $(1,4)$. BRAF mutational status has been established as routine in advanced malignant melanoma, demonstrated worse prognosis in patients harboring BRAF mutations (17).

Previous studies have shown that BRAF mutations correlated with primary tumor type, stage, thickness, and pronounced infiltration of lymphocytes $(18,19)$. However, some studies demonstrated that BRAF mutational status is not associated with disease-free survival in stage I and II melanoma (20). Because of the conflicting conclusions on BRAF mutations in the natural course of non-metastatic melanoma, their prognostic significance is still controversial.

The aim of the current study was to compare the value of the assessment of tumor-infiltrating lymphocytes (TIL) in stages I-II nodular and superficial spreading melanoma and BRAF mutational status, and its associations with clinicopathological characteristics.

\section{Patients and methods}

Design of the study. Eighty-five patients who underwent melanoma surgical treatment at Riga East University Hospital, Riga, Latvia, in 2012-2017 stage IA-IIC were retrospectively enrolled in the study. Only patients with nodular and superficial spreading melanoma were enrolled in this study.

Ethics. The study protocol was approved by the Central Medical Ethics Committee of Latvia (no. 01-29.1/2016-1-1 from January 2016) and the Ethical Committee of Institute of Cardiology and Regenerative Medicine, the University of Latvia (from September 2019; no. 12/2019). The study conducted according to the declaration of Helsinki and Oviedo Convention. All patients signed informed consent to participate in the study.

Exclusion criteria. Patients with lentigo maligna and acral lentiginous melanomas as well as patients who had stage III and IV melanoma, a previous history of cutaneous melanoma, uveal melanoma, mucosal melanoma, melanoma in situ, insufficient tumor and peritumoral tissue for histopathological examination, or who had undergone neoadjuvant treatment were excluded.

Clinical characteristics. Various clinical factors-age, gender, length of follow-up after surgery, recurrence, or metastasiswere obtained from medical records. Progression-free survival time was estimated from the surgical resection date to the first loco-regional or systemic metastasis or death without any type of relapse.
Histopathological characteristics. Whole section slides of tissues stained with hematoxylin and eosin (H\&E) were reviewed by two expert pathologists (T.Z. and S.I.) according to the current WHO (World Health Organization) and CAP (College of American Pathologists) guidelines (8). The pathological characteristics, including histological tumor subtype, Clark level, Breslow thickness, solar elastosis, ulceration, regression, lymphovascular invasion, and mitotic counts were evaluated.

Evaluation and scoring of peritumoral lymphocytes. Peritumoral lymphocytes were defined as lymphocytes surrounding the tumor mass. The lymphocyte distribution score was assessed. The lymphocyte distribution score, which ranged from 0 to 3 , was defined as follows: $0=$ absence of lymphocytes within the tissue, $1=$ presence of lymphocytes occupying $<25 \%$ of the tissue, $2=$ presence of lymphocytes occupying 25 to $50 \%$ of the tissue, and $3=$ presence of lymphocytes occupying $>50 \%$ of tissue. The low TIL infiltration was defined as scores 0 and 1 . The high TIL infiltration was defined as scores 2 and 3 .

BRAF mutation evaluation. Genomic DNA was isolated from $10 \mu \mathrm{m}$ sections cuts from formalin-fixed paraffin-embedded tissues using GeneRead ${ }^{\mathrm{TM}}$ DNA FFPE kit (Qiagen). The melanoma BRAF mutation status was assessed by digital droplet PCR (ddPCR) using BRAFV600 Screening Assay (Bio-Rad) as per the manufacturer's instructions. Droplets were generated using Biorad QX200 Droplet Generator and analyzed with QX200 Droplet Reader (Bio-Rad). Absolute quantifications of mutant and wild-type alleles were estimated by modeling a Poisson distribution using QuantaSoft ${ }^{\mathrm{TM}}$ analysis software version 1.7 (Bio-Rad).

Statistical analysis. The continuous values were reported as median (range). Differences in the expression levels of variables according to histopathologic and clinical characteristics were analyzed using the Chi-square, Mann-Whitney U test or the Kruskal-Wallis rank test. Association of mutation status with clinical and pathological features was analyzed by using Pearson $\chi^{2}$, Kruskal-Wallis test followed by Dunn's post-test together with the Log-logistic regression test to calculate statistical significance. Progression-free survival (PFS) was estimated with the Kaplan-Meier method with the log-rank test. Multivariate regression was analyzed using Cox proportional hazards model. Time was defined as the interval between the date of diagnosis and the date of disease recurrence (event) or last follow-up visit (censored).

Statistical calculations were performed with SPSS version 21.0 (IBM, Inc.). P-values of $<0.05$ were considered statistically significant.

\section{Results}

General characteristics. Altogether, 85 patients were enrolled in the study. 10 patients had stage IA, 13 patients had stage IB, 12 patients had stage IIA, 19 patients had stage IIB, and 31 patients had stage IIC melanoma. The median age was 66 years (range 24-84). 32 patients were males and 53 patients were females. Primary tumor localization was 
Table I. Clinicopathological characteristics of patients in the present study.

\begin{tabular}{|c|c|c|c|}
\hline Variables & $\begin{array}{l}\text { Nodular melanoma } \\
\qquad(\mathrm{n}=56)\end{array}$ & $\begin{array}{l}\text { Superficial spreading melanoma } \\
\qquad(\mathrm{n}=29)\end{array}$ & P-value \\
\hline Median age, years, n (range) & $67(27-84)$ & $64(24-83)$ & 0.230 \\
\hline Sex, male/female patients & $23 / 33$ & $9 / 20$ & $0.008^{\mathrm{a}, \mathrm{c}}$ \\
\hline Median Breslow thickness, mm, $\mathrm{n}$ (range) & $3.0(0.1-19.9)$ & $1.3(0.1-14.9)$ & $0.040^{\mathrm{b}, \mathrm{c}}$ \\
\hline Median Clark level, n (range) & $3(2-5)$ & $3(1-5)$ & $0.040^{\mathrm{b}, \mathrm{c}}$ \\
\hline Ulceration, present/absent & $41 / 15$ & $10 / 19$ & $0.010^{\mathrm{a}, \mathrm{c}}$ \\
\hline LVI, present/absent & $47 / 9$ & $21 / 8$ & 0.520 \\
\hline Neurotropism, present/absent & $4 / 52$ & $4 / 25$ & 0.720 \\
\hline Solar elastosis, $\mathrm{n}$ (range) & $2(0-3)$ & $1(0-3)$ & 0.320 \\
\hline Median tumor size, $\mathrm{cm}, \mathrm{n}$ (range) & $1.7(0.4-6.6)$ & $1.2(0.2-6.1)$ & $0.002^{\mathrm{b}, \mathrm{c}}$ \\
\hline Median mitotic count, $10 \mathrm{HPF}, \mathrm{n}$ (range) & $4(1-7)$ & $2(1-4)$ & $0.001^{\mathrm{b}, \mathrm{c}}$ \\
\hline Number of patients with low grade TIL, $n$ & 31 & 8 & $0.004^{\mathrm{a}, \mathrm{c}}$ \\
\hline TIL low grade, score $0-1$, mean (range) & $0.4(0.0-1.0)$ & $0.9(0.0-1.0)$ & $0.040^{\mathrm{b}, \mathrm{c}}$ \\
\hline Number of patients with high grade TIL, $n$ & 25 & 21 & 0.620 \\
\hline TIL high grade, score 2-3, mean (range) & $2(2-3)$ & $2(2-3)$ & 0.200 \\
\hline BRAF mutational status, V600 mutant/wild-type & $35 / 21$ & $17 / 12$ & 0.580 \\
\hline
\end{tabular}

${ }^{\mathrm{a} C h i}$-squared test; ${ }^{\mathrm{b}}$ Mann-Whitney U test; ${ }^{\mathrm{c}} \mathrm{P}<0.05$. LVI, lymphovascular invasion; HPF, high powered field; TIL, tumor infiltrating lymphocyte.

head/neck, limbs, and trunk in $16.1,38.7$ and $38.7 \%$ of patients, respectively.

Clinicopathological characteristics of patients with nodular and superficial spreading melanoma. There were 56 cases of nodular melanoma and 29 cases with superficial spreading melanoma (Table I). Nodular melanoma compared to the superficial spreading melanoma had a higher Clark level $(\mathrm{P}=0.04)$, greater Breslow thickness $(\mathrm{P}=0.04)$, more frequent ulceration $(\mathrm{P}=0.01)$, a higher mitotic count $(\mathrm{P}<0.0001)$ and greater tumor size $(\mathrm{P}=0.002)$. The nodular melanoma was more frequently found in males compared to females $(\mathrm{P}=0.008)$.

The lymphovascular invasion, neurotropism, and solar elastosis did not significantly differ between the groups. However, the correlation between the solar elastosis and Clark level was observed when all patients were analyzed together $\left(\chi^{2}=0.08 ; \mathrm{P}=0.02\right)$.

TIL in patients with nodular and superficial spreading melanoma. Peritumoral lymphocytes (TILs) were identified in 76 patients $(89.4 \%)$. The representative microphotograph of TILs in melanoma was presented in Fig. 1. The patients with nodular melanoma had predominantly low-grade TIL infiltration (score 1) compared to patients with superficial spreading melanoma ( $\mathrm{P}=0.004$; Table I).

A significant correlation between TIL score (both low and high $)$ and female gender $\left(\mathrm{R}^{2}=0.06 ; \mathrm{P}=0.025\right)$ and tumor size $\left(\chi^{2}=0.02 ; \mathrm{P}=0.005\right)$ has been observed in nodular melanoma and between TILs and mitotic count $\left(\chi^{2}=0.02 ; \mathrm{P}=0.018\right)$ in superficial spreading melanoma (Table II).

BRAF mutational status and its correlation with clinicopathological characteristics. All tissues were analyzed for BRAF mutational status (Fig. 2. BRAF V600 mutation was
Table II. Association analysis of tumor infiltrating lymphocytes with clinicopathological characteristics.

A, Superficial spreading melanoma

\begin{tabular}{lcc}
\hline Variables & P-value & $\chi^{2}$ value \\
\hline Age, years & 0.100 & N/A \\
Female patients & 0.200 & N/A \\
Breslow thickness & 0.200 & N/A \\
Clark level & 0.300 & N/A \\
Ulceration & 0.100 & N/A \\
LVI & 0.100 & N/A \\
Solar elastosis & 0.200 & N/A \\
Median tumor size & 0.400 & N/A \\
Median mitotic count & $0.020^{\mathrm{a}}$ & 0.02 \\
\hline
\end{tabular}

B, Nodular melanoma

\begin{tabular}{lll}
\hline Age, years & 0.800 & N/A \\
Female patients & $0.020^{\mathrm{a}}$ & 0.06 \\
Breslow thickness & 0.100 & $\mathrm{~N} / \mathrm{A}$ \\
Clark level & 0.500 & $\mathrm{~N} / \mathrm{A}$ \\
Ulceration & 0.090 & $\mathrm{~N} / \mathrm{A}$ \\
LVI & 0.200 & $\mathrm{~N} / \mathrm{A}$ \\
Solar elastosis & 0.500 & $\mathrm{~N} / \mathrm{A}$ \\
Median tumor size & $0.005^{\mathrm{a}}$ & 0.02 \\
Median mitotic count & 0.500 & $\mathrm{~N} / \mathrm{A}$ \\
\hline
\end{tabular}

${ }^{a} \mathrm{P}<0.05$. LVI, lymphovascular invasion; N/A, not applicable. Pearson's Chi-squared test $\left(\chi^{2}\right)$ was performed. 


\section{A}

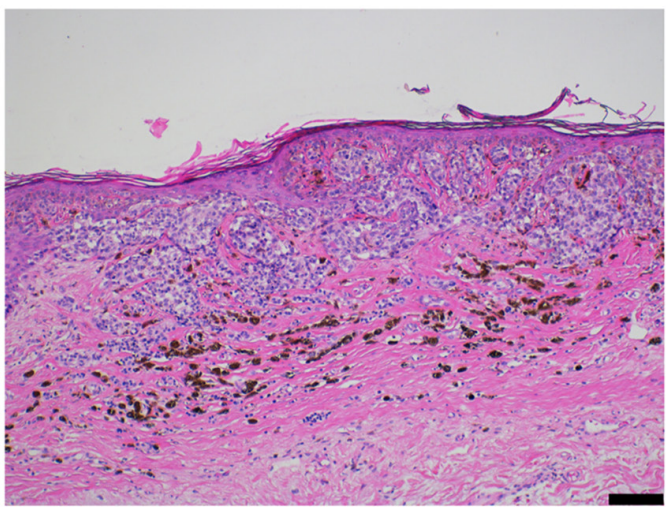

C

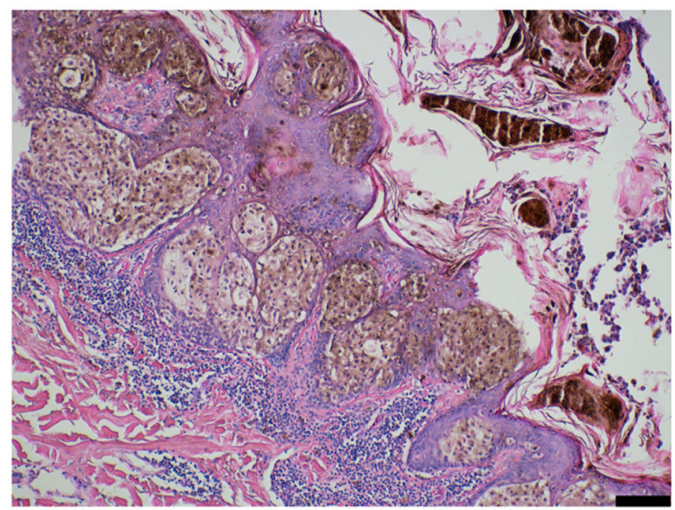

B

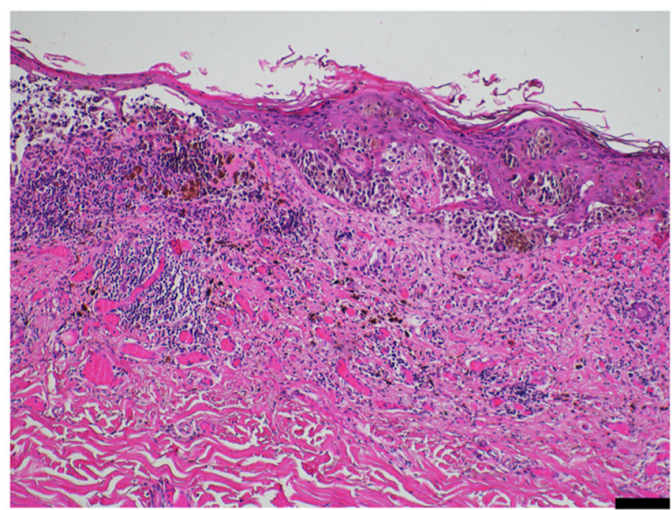

D

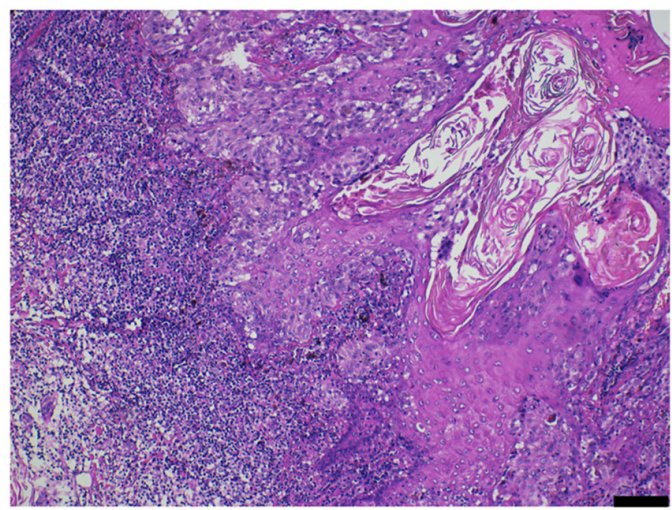

Figure 1. Representative photomicrograph demonstrating TILs in malignant melanoma. (A) Absent TIL infiltration, score 0. (B) Mild TIL infiltration, score 1. (C) Moderate TIL infiltration, score 2. (D) Prominent TIL infiltration, score 3. Hematoxylin and eosin staining method (magnification, $\mathrm{x} 100$; scale bar, $100 \mu \mathrm{m}$ ). TIL, tumor-infiltrating lymphocyte.

found in 52 out of 85 patients $(61.2 \%)$. The BRAF mutational status was associated with younger patient age $(\mathrm{P}=0.002)$. The associations of BRAF V600 mutational status and Clark invasion level, Breslow thickness, lymphovascular invasion, female gender and TIL was revealed (Table III). However, the correlation between the disease stage, mitotic activity, ulceration, median tumor size and BRAF mutational status was not demonstrated (Table III).

TIL, BRAF mutation status and progression-free survival. All 85 patients were clinically followed up, and there were 27 incidences of locoregional recurrence or systemic metastasis. When patient's progression-free survival (PFS) was compared based on the presence of TILs (both low or high grade), the patients with high TILs had significantly better prognosis compared to patients with low TIL grade $(\mathrm{HR}=4.9$, 95\% CI=2.3-11.2, $\mathrm{P}<0.0001$ ) (Fig. 3).

The PFS did not differ between wild-type and BRAF mutant melanoma $(\mathrm{HR}=1.8 ; 95 \% \mathrm{CI}=0.6-5.3, \mathrm{P}=0.28)$.

\section{Discussion}

Conventional prognostic characteristics of melanoma include melanoma type, lymphovascular invasion, ulceration, stage, Breslow thickness and mitotic rate, all of which are currently mandatory histopathological assessed criteria in all primary melanomas (8). TIL produced by the immune system in response to the invasion by the tumor, are frequently observed in tumor microenvironment, including those associated with cutaneous melanoma (9-11,21-24).
However, there is an unresolved debate in the literature whether the TIL grade is robust enough as a prognostic feature to be included in AJCC staging (8). Immune measures, especially TIL grade, are not standard components for melanoma pathology reports because they have not yet been validated and appreciated to impact overall survival or clinical management significantly (8). Furthermore, the association of TIL with an improved prognosis in melanoma remains controversial (9,22-29).

Different histopathological approaches for grading TILs have been described (11,21-22,24-28). Firstly, Clark's method graded TILs as absent, non-brisk, and brisk (22). Other studies graded TILs on a scale of 0 to 3 or only consider TILs as 'present' or 'absent' (25-29). Some studies use a combined grading system by assessing TIL density and distribution by a final score from 0 to 6 (11).

Previous studies have focused on the prognostic significance of TILs in primary melanoma $(9,22-29)$. However, the results are controversial. Several studies did not demonstrated the association between TIL presence and disease-free survival (23). Other studies showed a significant correlation between the TIL infiltration and patients' survival $(9,22,24,28-30)$.

Our study showed that high TIL infiltration in primary Stage I-II melanoma corresponded to a better prognosis, while low TIL infiltration demonstrated worse PFS. Recently, it was shown that brisk TIL grade is a favorable prognostic factor in primary melanomas and is a distinct group from non-brisk TIL grade based on disease progression and immunoregulatory gene expression profiles (30). Besides, TIL grade is an independent predictor of survival and SLN status in patients with melanoma. Patients with a pronounced TIL infiltrate 

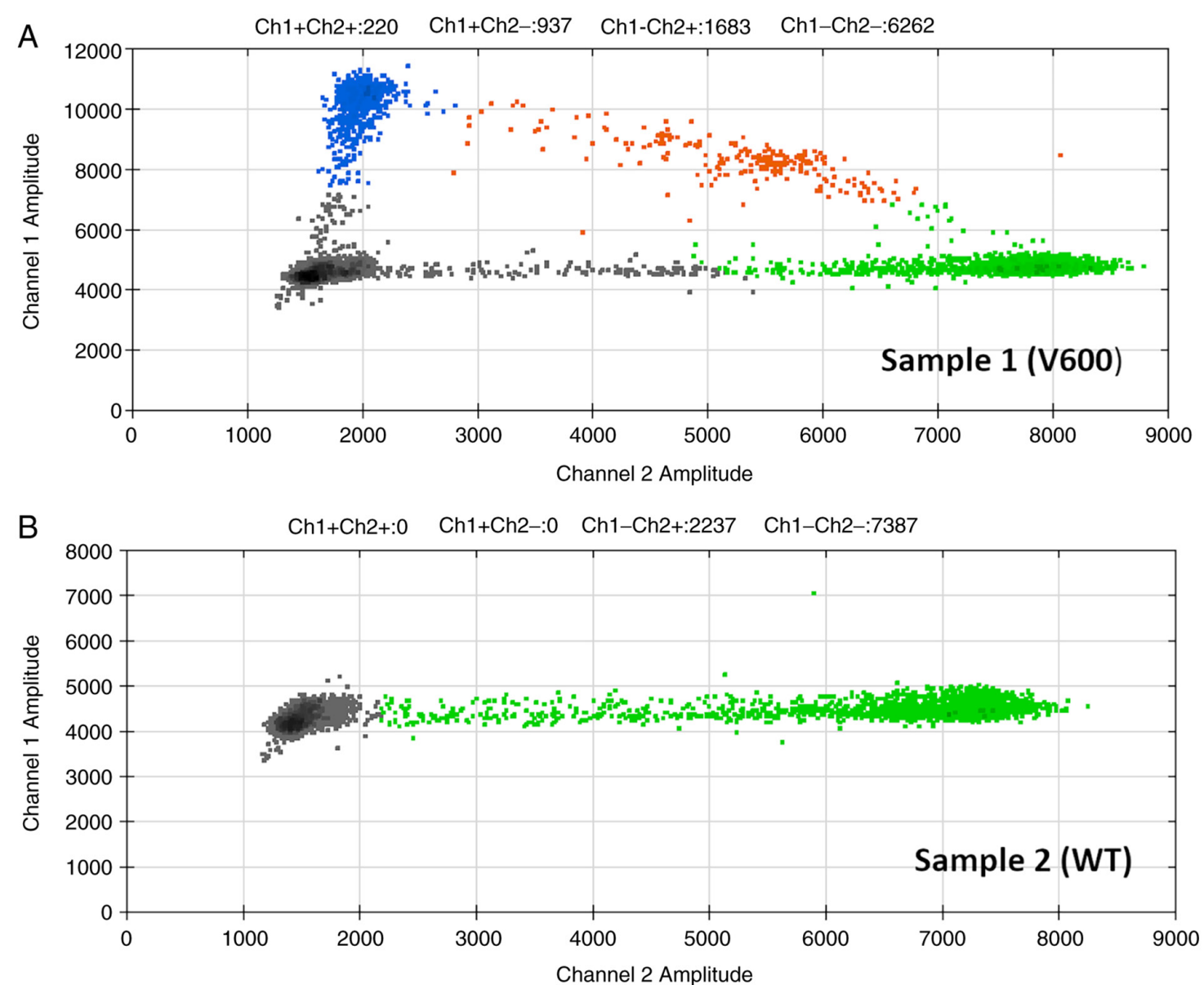

Figure 2. Two-dimensional Droplet Digital PCR plots. Plots of representative (A) BRAF V600 mutant and (B) WT tumors. WT, wild-type.

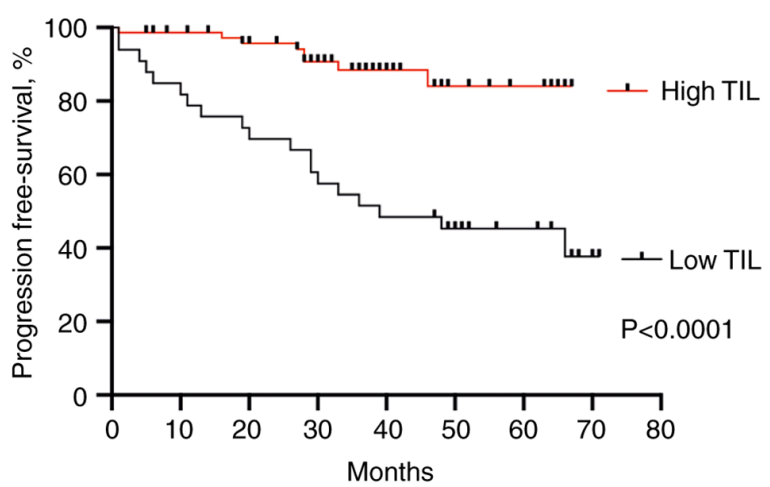

Figure 3. Progression-free survival in melanoma with high and low TIL infiltration. Kaplan-Meier plot was analyzed using a log-rank (Mantel-Cox) test. $\mathrm{P}<0.0001$. TIL, tumor-infiltrating lymphocyte.

overall have a better prognosis (9), and high levels of TILs in primary invasive melanoma are associated with favorable survival outcomes (10-12).

Our results were consistent with a recent study demonstrated that coexistence of tumor regression and TIL is associated with more favorable survival in melanoma (31).

However, in our study the association between tumor regression and TIL has not been demonstrated. This could be explained that in our study only patients with stage IA-IIC were enrolled.
Table III. Association analysis of BRAF mutation with clinicopathological characteristics.

\begin{tabular}{lcc}
\hline Variables & Pearson's $\chi^{2}$ value & P-value \\
\hline Age, years & 0.12 & 0.002 \\
Female patients & 0.09 & $0.009^{\mathrm{a}}$ \\
Breslow thickness & 0.07 & $0.020^{\mathrm{a}}$ \\
Clark level & 0.11 & $0.005^{\mathrm{a}}$ \\
Ulceration & 0.03 & 0.150 \\
LVI & 0.07 & $0.020^{\mathrm{a}}$ \\
Solar elastosis & 0.03 & 0.200 \\
Median tumor size & 0.01 & 0.800 \\
Median mitotic count & 0.06 & $0.040^{\mathrm{a}}$ \\
TIL & 0.06 & $0.040^{\mathrm{a}}$
\end{tabular}

${ }^{\mathrm{a}} \mathrm{P}<0.05$. LVI, lymphovascular invasion; TIL, tumor infiltrating lymphocyte.

In our study, the patients with nodular melanoma demonstrated downregulation of TIL compared to superficial spreading melanoma. Previous studies have demonstrated decreased TILs in nodular melanomas compared with matched superficial spreading ones (32). However, this observation was found only in thin $(\leq 2 \mathrm{~mm})$ melanomas and in 
males' patients (32). These findings suggested the differences in immunological response between the gender and tumor thickness. Furthermore, the tumor stage and immunologic heterogeneity of tumor-infiltrating lymphocyte subpopulation could contribute to it (30).

BRAF mutations in primary melanomas are seen at a rate of $22-72 \%$ (30). In our study, BRAF mutation was found in $61.2 \%$ of all cases analyzed.

Previous studies showed the different value of BRAF mutational status in association with clinicopathological characteristics and PFS of melanoma (30-36).

It has been demonstrated that BRAF mutational status correlate with younger age and female gender (33).

Age is an independent negative predictor of melanoma survival (32). Nonetheless, the melanoma association with gender appears to be controversial. There are studies demonstrating associations with the male gender (33) and other studies showing associations with the female gender (34). Varying patterns of immunological responses might be involved in the age and gender differences of melanoma prognosis (30).

Previous studies demonstrated the association of BRAF mutational status and tumor ulceration, solar elastosis, advanced melanoma stage and histologic subtype $(36,37)$.

At contrast, in young patients the study showed that there was no significant association between BRAF mutational status and gender, histologic subtype, the Clark and Breslow level, solar elastosis and lymphovascular invasion, suggested different tumorigenesis pathways in younger population (38).

The results of our study are consistent with previous findings and extent these by demonstrating that both Clark and Breslow level correlated with BRAF mutational status. In addition, the association of BRAF mutational status and vascular invasion and TIL has been observed. This observation led us to the suggestion that stage I-II BRAF mutant melanoma compared to wild-type characterized by more invasive pattern.

Some previous studies have shown the association of BRAF mutational status and solar elastosis (35). Although, the correlation between BRAF mutational status and solar elastosis was not observed in our study. The geographical characteristics and differences in sun exposure could contribute to it.

Some previous studies also demonstrate the association of BRAF mutational status and ulceration $(35,36)$, while other analyses did not observe a significant association (37).

The association of BRAF mutational status with ulceration was not observed in our study. It could be suggested that ulceration in larger tumors could be related to BRAF mutational status, however the median tumor size was smaller compared to previous studies $(35,36)$.

The value of BRAF mutational status and TIL has been addressed in several studies, however, the results were controversial. Our study showed that TIL was upregulated in BRAF mutant melanoma compared to BRAF wild-type. However, some recent studies did not found associations between TIL and BRAF mutational status $(35,38,39)$.

At contrast, it has been demonstrated that the intratumoral density of TIL was significantly higher in BRAF mutant melanoma (40). Intratumor heterogeneity of melanoma with different cytokine milieu and expression of immunomodulatory genes might contribute to it. In addition, a recent study has demonstrated that higher TIL clusters are associated with response to immunotherapy in BRAF $\mathrm{V} 600 \mathrm{E} / \mathrm{K}$ mutated melanoma (41).

Since BRAF inhibitors constitute a cornerstone in the treatment of advance melanoma, the role of BRAF mutational status of PFS is of particular importance.

A recent study showed that patients with localized BRAF-mutant melanomas experienced worse PFS than those with BRAF wild-type $(42,43)$. However, our study and some previous research did not found significant association between PFS and BRAF mutational status in stage I-II melanoma (18-20). The different study population, patient age, gender, as well as tumor heterogeneity could contribute to it.

Several limitations of our study should be mentioned. A significantly higher number of case-cohort is required to clearly show whether these associations identified by many studies, including ours, are influenced by the variance of different populations, disease stage, age, and melanoma types presented in each study. At the same time, the strength of the present study was the demonstration of significant role of TIL and BRAF mutational status in patients with stage I-II melanoma. In addition, the study design included the patients from Ria East University Hospital, which served up to $85 \%$ of all melanoma cases in Latvia.

To conclude, the patients with nodular melanoma had predominantly low-grade TIL infiltration compared to patients with superficial spreading melanoma. In addition, high TIL infiltration corresponded to better prognosis and correlated with BRAF mutational status.

The assessment of TILs in melanoma proved prognostic significance value should be performed in routine histopathological examination.

\section{Acknowledgements}

The authors would like to acknowledge Mrs Aija Ozola and Mr Mohamed Omar from the Latvian Biomedical Research and Study Centre (Riga, Latvia) for their valuable input in BRAF genetic testing and Dr Kristine Vaivode from the Latvian Biomedical Research and Study Centre for language editing the manuscript.

\section{Funding}

The present study was supported by a PhD student grant from the Faculty of Medicine of the University of Latvia (grant no. 1.1.1.1/20/A/146) and a grant from the European Regional Development Fund (grant no. 1.1.1.1/18/A/099).

\section{Availability of data and materials}

The datasets used and/or analyzed during the current study are available from the corresponding author on reasonable request.

\section{Authors' contributions}

All authors have contributed to and agreed on the content of the manuscript. TZ, AN and SI analyzed the histopathological slides and data. DP and MK established the mutation-screening system and performed genetic analysis. TZ, SI and DP wrote the manuscript. TZ, AN and SI participated in the patient 
enrollment, and data collection and analysis. SI and DP supervised the project. All authors have read and approved the final manuscript. TZ, SI and DP confirm the authenticity of all the raw data.

\section{Ethics approval and consent to participate}

The study protocol was approved by the Central Medical Ethics Committee of Latvia (approval no. 01-29.1/2016-1-1; January 2016) and the Ethical Committee of the Institute of Cardiology and Regenerative Medicine, University of Latvia (approval no. 12/2019; September 2019; Riga, Latvia). The study was conducted according to the Declaration of Helsinki and Oviedo Convention. All subjects signed informed consent to participate in the study.

\section{Patient consent for publication}

Not applicable.

\section{Competing interests}

The authors declare that they have no competing interests.

\section{References}

1. Hessler M, Jalilian E, Xu Q, Reddy S, Horton L, Elkin K, Manwar R, Tsoukas M, Mehregan D and Avanaki K: Melanoma biomarkers and their potential application for in vivo diagnostic imaging modalities. Int J Mol Sci 21: 9583, 2020.

2. Forsea AM: Melanoma epidemiology and early detection in Europe: Diversity and disparities. Dermatol Pract Concept 10: e2020033, 2020

3. Shellenberger R, Nabhan M and Kakaraparthi S: Melanoma screening: A plan for improving early detection. Ann Med 48 $142-148,2016$

4. Mandalà $\mathrm{M}$ and Massi $\mathrm{D}$ : Tissue prognostic biomarkers in primary cutaneous melanoma. Virchows Arch 464: 265-281, 2014.

5. Romaine ST, Wells-Jordan P, de Haro T, Dave-Thakrar A, North J, Pringle JH and Saldanha G: A small multimarker panel using simple immunohistochemistry methods is an adjunct to stage for cutaneous melanoma prognosis. Melanoma Res 26 : $580-587,2016$

6. Bolognia J, Schaffer J and Cerroni L (eds): Dermatology. Elsevier, Amsterdam, 2018

7. Bastian BC: The molecular pathology of melanoma: An integrated taxonomy of melanocytic neoplasia. Annu Rev Pathol 9 : 239-271, 2014.

8. Elder DE, Massi D, Scolyer RA and Willemze R (eds): WHO Classification of Skin Tumours, WHO, 2018.

9. Azimi F, Scolyer RA, Rumcheva P, Moncrieff M, Murali R, McCarthy SW, Saw RP and Thompson JF: Tumor-infiltrating lymphocyte grade is an independent predictor of sentinel lymph node status and survival in patients with cutaneous melanoma. J Clin Oncol 30: 2678-2683, 2012.

10. Fortes C, Mastroeni S, Mannooranparampil TJ, Passarelli F, Zappala A, Annessi G, Marino C, Caggiati A, Russo N and Michelozzi P: Tumor-infiltrating lymphocytes predict cutaneous melanoma survival. Melanoma Res 25: 306-311, 2015.

11. Park CK and Kim SK: Clinicopathological significance of intratumoral and peritumoral lymphocytes and lymphocyte score based on the histologic subtypes of cutaneous melanoma. Oncotarget 8: 14759-14769, 2017.

12. Maibach F, Sadozai H, Seyed Jafari SM, Hunger RE and Schenk M: Tumor-infiltrating lymphocytes and their prognostic value in cutaneous melanoma. Front Immunol 11: 2105, 2020.

13. Dougan M, Dranoff G and Dougan SK: Cancer immunotherapy: Beyond checkpoint blockade. Annu Rev Cancer Biol 3: 55-75, 2018

14. Havel JJ, Chowell D and Chan TA: The evolving landscape of biomarkers for checkpoint inhibitor immunotherapy. Nat Rev Cancer 19: 133-150, 2019.
15. Ribas A and Wolchok JD: Cancer immunotherapy using checkpoint blockade. Science 359: 1350-1255, 2015.

16. Cancer Genome Atlas Network: Genomic classification of cutaneous melanoma. Cell 161: 1681-1696, 2015.

17. Ny L,Hernberg M, Nyakas M, Koivunen J, Oddershede L, Yoon M, Wang X, Guyot P and Geisler J: BRAF mutational status as a prognostic marker for survival in malignant melanoma: A systematic review and meta-analysis. Acta Oncol 59: 833-844, 2020.

18. Edlundh-Rose E, Egyhazi S, Omholt K, Mansson-Brahme E, Platz A, Hansson J and Lundeberg J: NRAS and BRAF mutations in melanoma tumours in relation to clinical characteristics: A study based on mutation screening by pyrosequencing. Melanoma Res 6: 471-478, 2006.

19. Eigentler T, Assi Z, Hassel JC, Heinzerling L, Starz H, Berneburg M, Bauer J and Garbe C: Which melanoma patient carries a BRAF-mutation? A comparison of predictive models. Oncotarget 7: 36130-36137, 2016.

20. Tas F and Erturk K: Clinical and prognostic significance of BRAF V600E mutation in non-metastatic cutaneous melanoma patients. Neoplasma 66: 631-636, 2019.

21. Sun Q, Sun H, Wu N, Cong L and Cong X: Prognostic significance of tumor-infiltrating lymphocyte grade in melanoma: A meta-analysis. Dermatology 236: 481-492, 2020.

22. Clark WH Jr, Elder DE, Guerry D IV, Braitman LE, Trock BJ, Schultz D, Synnestvedt M and Halpernet AC: Model predicting survival in stage I melanoma based on tumor progression. J Natl Cancer Inst 81: 1893-1904, 1989.

23. Barnhill RL, Fine JA, Roush GC and Berwick M: Predicting five-year outcome for patients with cutaneous melanoma in a population-based study. Cancer 78: 427-432, 1996.

24. Tuthill RJ, Unger JM, Liu PY, Flaherty LE and Sondak VK; Southwest Oncology Group: Risk assessment in localized primary cutaneous melanoma: A Southwest Oncology Group study evaluating nine factors and a test of the Clark logistic regression prediction model. Am J Clin Pathol 118: 504-511, 2002.

25. Taylor RC, Patel A, Panageas KS, Busam KJ and Brady MS: Tumor-infiltrating lymphocytes predict sentinel lymph node positivity in patients with cutaneous melanoma. J Clin Oncol 25: 869-875, 2007.

26. Burton AL, Roach BA, Mays MP, Chen AF, Ginter BA, Vierling AM, Scoggins CR, Martin RC, Stromberg AJ, Hagendoorn L and McMasters KM: Prognostic significance of tumor infiltrating lymphocytes in melanoma. Am Surg 77: 188-192, 2011.

27. Mandala M,Imberti GL, Piazzalunga D, Belfiglio M, Labianca R, Barberis M, Marchesi L, Poletti P, Bonomi L, Novellino L, et al: Clinical and histopathological risk factors to predict sentinel lymph node positivity, disease-free and overall survival in clinical stages I-II AJCC skin melanoma: Outcome analysis from a single-institution prospectively collected database. Eur J Cancer 45: 2537-2545, 2009.

28. Thomas NE, Busam KJ, From L, Kricker A, Armstrong BK, Anton-Culver H, Gruber SB, Gallagher RP, Zanetti R, Rosso S, et al: Tumor-infiltrating lymphocyte grade in primary melanomas is independently associated with melanoma-specific survival in the population-based genes, environment and melanoma study. J Clin Oncol 31: 4252-4259, 2013.

29. Clemente CG, Mihm MC Jr, Bufalino R, Zurrida S, Collini P and Cascinelli N: Prognostic value of tumor infiltrating lymphocytes in the vertical growth phase of primary cutaneous melanoma. Cancer 77: 1303-1310, 1996.

30. Weiss SA, Han SW, Lui K, Tchack J, Shapiro R, Berman R, Zhong J, Krogsgaard M, Osman I and Darvishian F: Immunologic heterogeneity of tumor-infiltrating lymphocyte composition in primary melanoma. Hum Pathol 57: 116-125, 2016.

31. Tas F and Erturk K: Coexistence of regression and tumor infiltrating lymphocytes is associated with more favorable survival in melanoma. J Cancer Res Clin Oncol 147: 2721-2729, 2021.

32. Lin RL, Wang TJ, Joyce CJ, Mihm MC Jr, Murphy GF, Lian CG and Lin YE: Decreased tumor-infiltrating lymphocytes in nodular melanomas compared with matched superficial spreading melanomas. Melanoma Res 26: 524-527, 2016.

33. Platz A, Egyhazi S, Ringborg U and Hansson J: Human cutaneous melanoma; a review of NRAS and BRAF mutation frequencies in relation to histogenetic subclass and body site. Mol Oncol 1: 395-405, 2008

34. Yaman B, Akalin T and Kandiloğlu G: Clinicopathological characteristics and mutation profiling in primary cutaneous melanoma. Am J Dermatopathol 37: 389-397, 2015. 
35. Bezić J, Kuret S, Vrbičić B, Smolić J, Borić I, Škifić I, Ledina D and Božić J: Clinicopathological characteristics of BRAF V600E mutated melanomas in the dalmatian region of croatia. Acta Dermatovenerol Croat 27: 225-230, 2019.

36. Spathis A, Katoulis AC, Damaskou V, Liakou AI, Kottaridi C, Leventakou D, Sgouros D, Mamantopoulos A, Rigopoulos D, Karakitsos P and Panayiotides IG: BRAF mutation status in primary, recurrent, and metastatic malignant melanoma and its relation to histopathological parameters. Dermatol Pract Concept 9: 54-62, 2019.

37. Kim SY, Kim SN, Hahn HJ, Lee YW, Choe YB and Ahn KJ: Metaanalysis of BRAF mutations and clinicopathologic characteristics in primary melanoma. J Am Acad Dermatol 72: 1036-1046.e2, 2015.

38. Estrozi B, Machado J, Rodriguez $R$ and Bacchi CE: Clinicopathologic findings and BRAF mutation in cutaneous melanoma in young adults. Appl Immunohistochem Mol Morphol 22: 57-64, 2014.

39. Aksenenko MB, Kirichenko AK and Ruksha TG: Russian study of morphological prognostic factors characterization in BRAF-mutant cutaneous melanoma. Pathol Res Pract 211: 521-527, 2015.

40. Leslie C, Bowyer SE, White A, Grieu-Iacopetta F, Trevenen M, Iacopetta B, Amanuel B and Millward M: $\mathrm{FOXP}^{+} \mathrm{T}$ regulatory lymphocytes in primary melanoma are associated with BRAF mutation but not with response to BRAF inhibitor. Pathology 47 $557-563,2015$.
41. Klein S, Mauch C, Brinker K, Noh KW, Knez S, Büttner R, Quaas A and Helbigc D: Tumor infiltrating lymphocyte clusters are associated with response to immune checkpoint inhibition in BRAF V600 ${ }^{\mathrm{E} / \mathrm{K}}$ mutated malignant melanomas. Sci Rep 11: 1834, 2021.

42. Safaee Ardekani G, Jafarnejad SM, Khosravi S, Martinka M, Ho V and Li G: Disease progression and patient survival are significantly influenced by BRAF protein expression in primary melanoma. Br J Dermatol 169: 320-328, 2013.

43. Nagore E, Requena C, Traves V, Guillen C, Hayward NK, Whiteman DC and Hacker E: Prognostic value of BRAF mutations in localized cutaneous melanoma. J Am Acad Dermatol 70: 858-862.e1-2, 2014.

(†) $\Theta$ This work is licensed under a Creative Commons Attribution-NonCommercial-NoDerivatives 4.0 International (CC BY-NC-ND 4.0) License. 